\title{
Evaluation of a Non-Invasive Respiratory Volume Monitor Without Patient-Specific Calibration
}

Barak Cohen', Hani Essber', Logan Glosser', Joseph Vitale1, Jordan Brayanov², Alparslan Turan

'Outcomes Research, Anesthesiology Institute, Cleveland Clinic Foundation, Cleveland, OH, USA; ${ }^{2}$ Respiratory Motion Inc., Waltham MA, USA;

\section{Introduction}

- Continuous monitoring of respiratory status is important for identifying potentially life-threatening respiratory compromise, performing clinically appropriate interventions, and monitoring patient recovery.

- Recently, a non-invasively Respiratory Volume Monitor (RVM, ExSpiron, Respiratory Motion) was developed and has been shown to have better than $10 \%$ accuracy for minute ventilation (MV), tidal volume (TV), and respiratory (RR) when tested against spirometry and ventilator measurement (Anesth Analg. 2013 Jul;117(1):91-100; J Clin Monit Comput. 2015 Apr;29(2):223-30).

- The current RVM requires a patient-specific, single-point calibration with a spirometer in order to make quantitative measurements of MV and TV.

- To facilitate broader use of the technology and eliminate error introduced by variability in the use of the spirometer itself, the RVM has been updated to make quantitative measurements of MV and TV without the need for a patient-specific calibration.

- Objective: Evaluate the accuracy of the RVM without patient-specific calibration compared to three different FDA-cleared devices in healthy volunteers.

\section{Methods}

- MV, TV, and RR were simultaneously recorded by the RVM without Patient-Specific Calibration and an FDA-cleared Predicate Device.

- Two sets of breathing trials, separated by approximately $24 \mathrm{hr}$, were performed. Subjects kept the RVM electrode padset on between Day 1 and Day 2 trials.

- Each day consisted of three, 10 min trials with a different FDA-cleared device

- Pneumotachometer (Heated FVL, Morgan Scientific)

- Wright Spirometer (Mark 14, nSpire Health, Inc.)

- RVM with Patient-Specific Calibration (ExSpiron, Respiratory Motion).

- After the completion of the breathing trials, subjects kept the RVM electrode pads on and the same 3 breathing trials were repeated 24 hours later (Day 2).

- Measurement errors between the RVM without patient-specific calibration and the FDA-cleared devices were quantified over each 30 second segment on both an absolute and relative (i.e., Bland-Altman) basis.

- Paired-difference equivalence tests were performed with equivalence margins of $10 \%$ relative error.
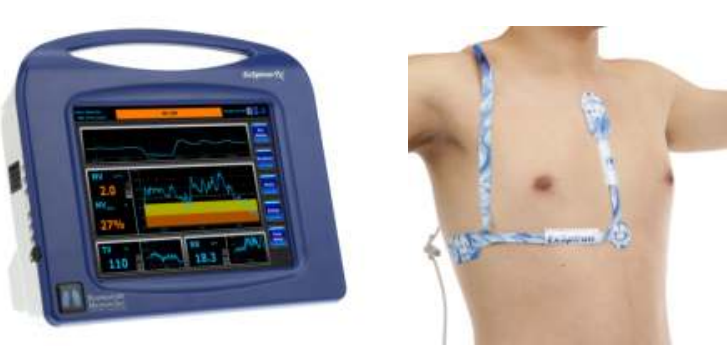

Figure 1. Non-invasive RVM that provides continuous, real-time measurements of MV TV, and RR. Figure shows standard PadSet placement: sternal notch, xiphoid, and right mid-axillary line at the level of the xiphoid.

\section{Results}

Table 1. Subject Cohort Anthropometrics

- Subjects varied in age, ethnicity, and anthropometrics.

- Pertinent co-morbidities included asthma, bronchitis, and obstructive sleep apnea.

\begin{tabular}{|c|c|c|c|}
\hline Anthropometrics & Mean & SD & Range \\
\hline Number of Patients & 20 & & \\
\hline Males / Females & $9 / 11$ & & \\
\hline Age, years & 49.2 & 17.5 & $22-80$ \\
\hline Weight, kg & 76.0 & 18.9 & $44.9-117.5$ \\
\hline Height, cm & 167.8 & 10.7 & $152-185$ \\
\hline BMI, kg/m & 26.8 & 5.6 & $18.7-41.8$ \\
\hline
\end{tabular}
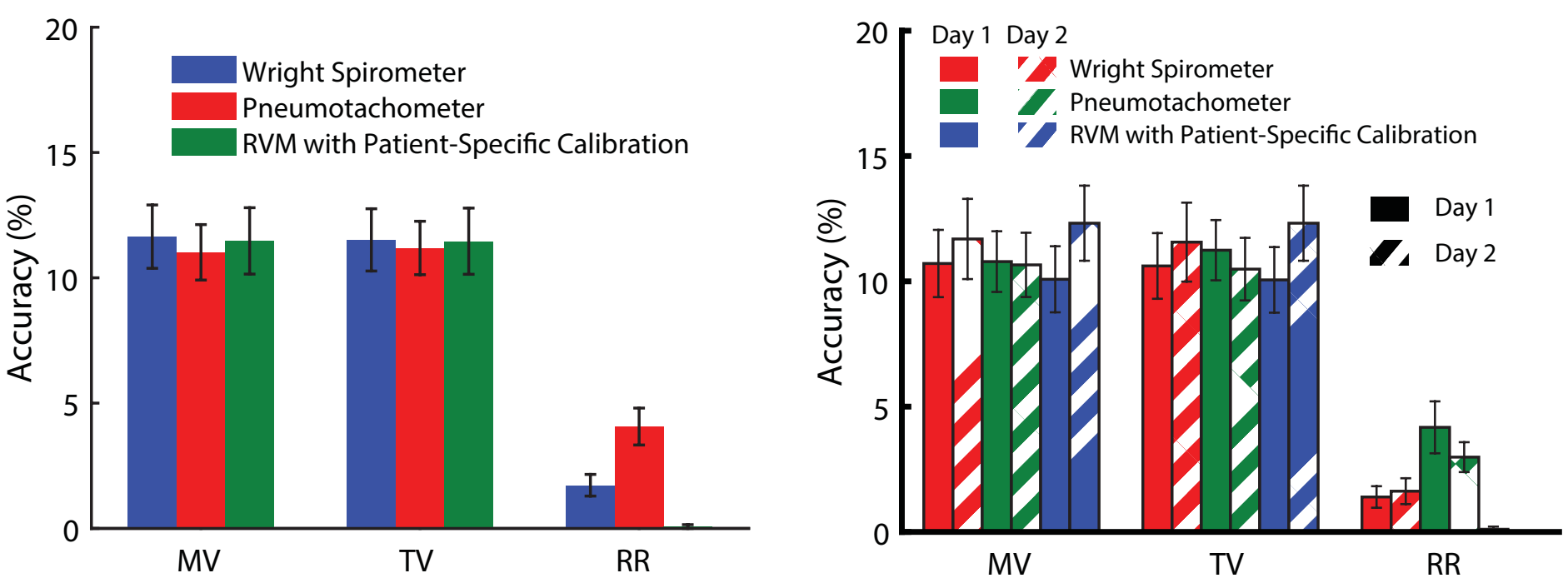

Figure 2. Mean accuracy of RVM without Patient-Specific Calibration compared to three FDA-cleared devices.

- The mean measurement accuracies were better than 11.6\% for MV (1.02 $\mathrm{L} / \mathrm{min})$, and TV $(81.9 \mathrm{~mL})$, and $4.1 \%$ (0.62 bpm) compared to the FDA-cleared devices.

- Measurement accuracies were not significantly different on Day 1 and Day 2 (Two-way ANOVAs, $\mathrm{p}>0.10$ ).

Table 2. Mean Bias, Precision, and Accuracy of RVM without patient-specific calibration compared to three FDA-cleared devices

\begin{tabular}{|l|c|c|c|c|c|c|c|c|c|}
\hline & \multicolumn{3}{|c|}{$\begin{array}{c}\text { Minute Ventilation, \% } \\
\text { (L/min) }\end{array}$} & \multicolumn{3}{c|}{$\begin{array}{c}\text { Tidal Volume, \% } \\
\text { (mL) }\end{array}$} & \multicolumn{3}{c|}{$\begin{array}{c}\text { Respiratory Rate, \% } \\
\text { (bpm) }\end{array}$} \\
\hline & Bias & Prec & Accu & Bias & Prec & Accu & Bias & Prec & Accu \\
\hline \multirow{2}{*}{ Wright Spirometer } & $-1.8 \%$ & $8.9 \%$ & $11.6 \%$ & $-1.5 \%$ & $8.8 \%$ & $11.5 \%$ & $-0.23 \%$ & $2.5 \%$ & $1.7 \%$ \\
& $(-0.30)$ & $(0.79)$ & $(1.02)$ & $(-24.6)$ & $(68.0)$ & $(81.9)$ & $(-0.03)$ & $(0.33)$ & $(0.23)$ \\
\hline Pneumotachometer & 2.2 & $7.7 \%$ & 11.0 & 2.2 & $7.8 \%$ & 11.2 & 0.00 & $5.1 \%$ & 4.1 \\
& $(0.08)$ & $(0.67)$ & $(0.97)$ & $(7.6)$ & $(49.3)$ & $(72.0)$ & $(0.01)$ & $(0.87)$ & $(0.62)$ \\
\hline RVM with Patient- & 2.2 & $8.1 \%$ & 11.5 & 2.2 & $8.1 \%$ & 11.4 & 0.01 & $0.3 \%$ & 0.1 \\
Specific Calibration & $(0.08)$ & $(0.66)$ & $(1.00)$ & $(7.4)$ & $(47.6)$ & $(76.4)$ & $(0.003)$ & $(0.08)$ & $(0.02)$ \\
\hline
\end{tabular}

Table 3. Paired-difference equivalence tests concluded that the measurements of MV, TV, and RR from the RVM without patient-specific calibration and each of the three Predicate Devices are equivalent within $\pm 10 \%$.

\begin{tabular}{|c|c|c|c|c|}
\hline Predicate Device & MV 90\% CI & TV, 90\% CI & TV, 90\% CI & Result \\
\hline Wright Spirometer & $(-6.3 \%, 3.2 \%)$ & $(-6.0 \%, 3.4 \%)$ & $(-1.5 \%, 1.1 \%)$ & Equivalent \\
\hline Pneumotachometer & $(-2.3 \%, 6.0 \%)$ & $(-2.3 \%, 6.1 \%)$ & $(-2.7 \%, 2.7 \%)$ & Equivalent \\
\hline RVM w/ Patient-Specific Calibration & $(-2.4 \%, 6.3 \%)$ & $(-2.4 \%, 6.3 \%)$ & $(-0.2 \%, 0.2 \%)$ & Equivalent \\
\hline
\end{tabular}

Figure 3. Measurement error analysis for 20 subjects.

- Left: Correlation plots

- X-axes: ExSpiron with Patient-Specific Calibration; Y-axes: FDA-Cleared Devices - $96.7 \%, 96.7 \%$, and $100 \%$ of patients are within the $20 \%$ relative error bounds (dashed lines) for MV, TV, and RR, respectively

- Right: Bland-Altman analysis

- X-axes: average measurement of ExSpiron without Patient-Specific Calibration and three Predicate Device; Y-axes: difference between device measurements
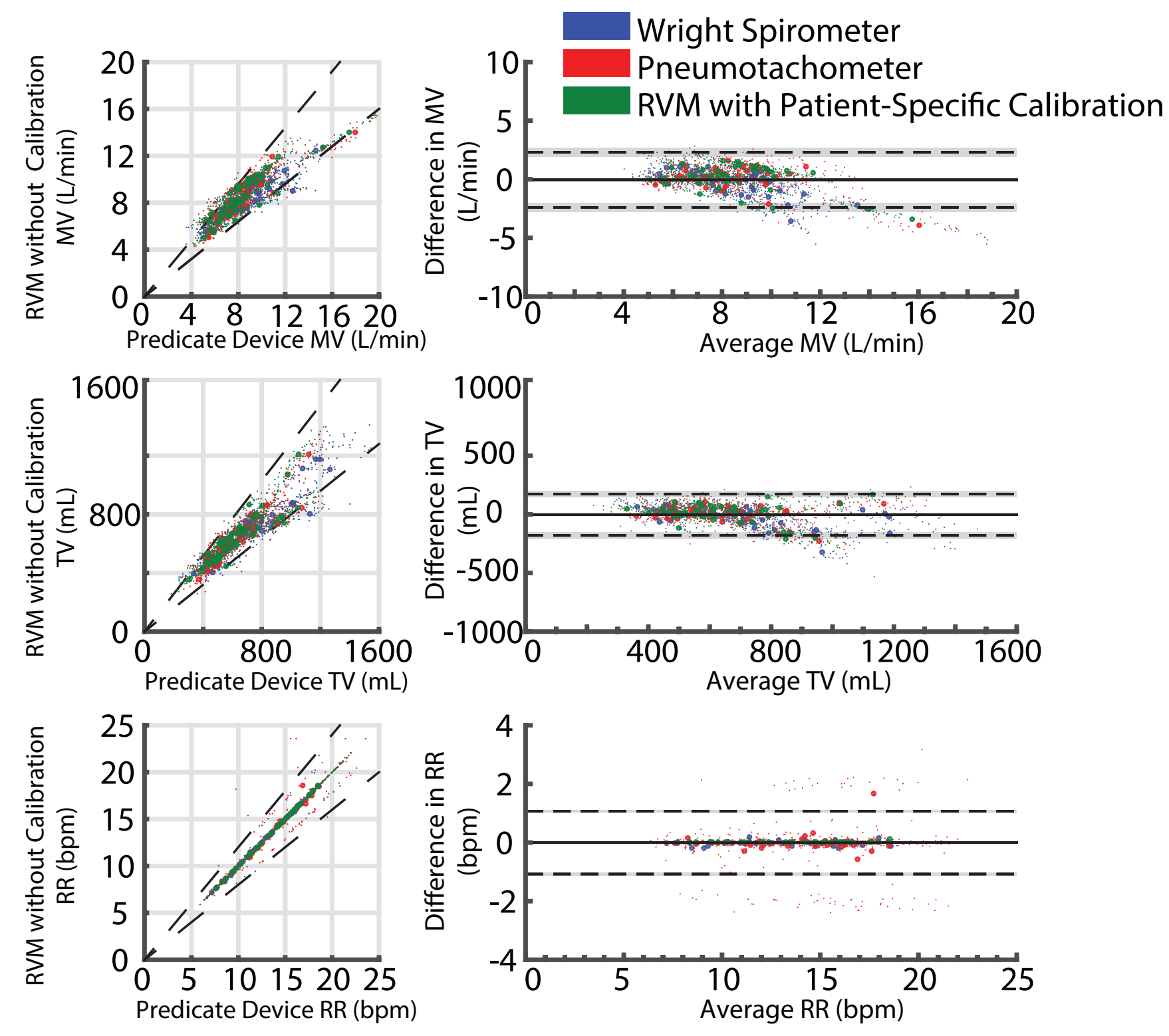

\section{Discussion}

- The updated RVM delivers clinically relevant measurements of MV, TV, and RR with $12 \%$ for MV and TV and 5\% (0.6 bpm) for RR without the need for a patient-specific calibration.

- Removing the need for calibration or baseline measurement eliminate error associated with the spirometer and expands the possible uses of the RVM to:

- Patients who were never on a mechanical ventilator

- Patients with suspected respiratory compromise

- Patients who have already received opioids or sedatives

- Monitor improvement or deterioration of an uncooperative patient in respiratory distress or failure

- Prehospital or home setting

- ICU patients to determine if there is a need to intubate

- Patients unable to perform spirometer calibration

- Patients being weaned off of a ventilator 